\title{
Editorial
}

\section{Adjuvant in central neuraxial blocks}

Central Neuraxial Block (CNB) occupies an important part in anaesthesia and pain management. Local anaesthetic agents block the generation and propagation of action potential in all excitable tissues primarily by impairing the function on sodium channels in the axonal membrane ${ }^{1}$. The complex neurophysiology of dorsal horn involves many neurotransmitters. These substances including substance $\mathrm{P}$, serotonin, acetylcholine, adenosine and glutamate are related in the dorsal horn and modulate peripheral nociceptive input ${ }^{2}$. A wide variety of adjuvant agents are used along with local anaesthetic mixtures to enhance and prolong their action, reduce unwanted motor weakness and autonomic dysfunction and reduce central nervous system and cardiovascular toxicity. The adjuvant drugs interact with one or more of these neurotransmitters exerting an anti-nociceptive effect. The most widely used drugs are opioid agonist like morphine, fentanyl, á 2 adrenergic agonist like clonidine, epinephrine, anti-cholinergic agent like neostigmine, NMDA receptor antagonist like ketamine and magnesium sulphate. Whatever the agent are used, it should be preservative free, nontoxic to neuron and should not contain any inhibitory neurotransmitters ${ }^{3}$.

Neuraxial administered opioid produce significant dose-dependent analgesia through opioid receptors is abundantly present in the substantia gelatinosa. It inhibits a voltage- sensitive calcium channel on presynaptic nerve terminals. This action inhibits release of neurotransmitters, including substance $P$ and glutamate that are active in spinal nociceptive transmission. Secondly, opioids can hyperpolarize and thus inhibit postsynaptic neurons by opening potassium channels ${ }^{4}$. Addition of opioid especially morphine and fentanyl is now routinely practiced world wide during central Neuraxial block. This practice is not popular in Bangladesh. It is probably due to lack of interest, availability of preservation free morphine and undue fear of respiratory depression.

Acetylcholine receptors are expressed in the dorsal horn. They contribute to the descending modulation of ascending nociceptive transmission. Neostigmine added to intrathecal bupivacaine has been shown to reduce postoperative analgesic requirements, improve sensory and motor block and delay resolution of block ${ }^{5}$. There is also some evidence that it can minimize the sympathetic blockade, reducing the hypotension that accompanies central neural local anaesthetic blockade. However, nausea and vomiting and faecal incontinence resistant to treatment preclude its use in current clinical practice in higher doses. .

Benzodiazepines receptors were first identified in the central nervous system in 1977. The highest concentration of receptors is found in the cerebral cortex, hypothalamus, cerebellum, corpus striatum and finally, medulla. Antinociceptive effect and safety of intrathecally-administered midazolam is well established ${ }^{6}$. Neuraxial administered magnesium sulphate also produce anti-nociccptive effects and it is currently used in both obstetric and non-obstetric population ${ }^{7}$. It is also used in labour analgesia as an adjuvant ${ }^{8}$.

\section{Professor AKM Akhtaruzzaman}

Department of Anaesthesia, Intensive Care and Pain Medicine, Bangabandhu Sheikh Mujib Medical University, Shahbag, Dhaka-1000; Email:<mili@ bol-online.com>; <akhtaruzzaman@bsmmu.org>

\section{References}

1. Butterworth JF, Strichartz GR. Molecular mechanism of local anaesthetic: a review. Anaesthesiology 1990; 72:711-34.

2. Yaksh TI, Huo XY, Kalcheva I, Nozaki TN, Marshala M. the spinal biology in human and animals of pain states generated by persistent small afferent input. Proc Natl Acad Sci 1999; 96:7680-6.

3. Shudhakar RM, Mathew RC. Adjuvant agents in regional anaesthesia. Anaesthesia and Intensive Care Medicine 2009; 10:538-40. 
4. Woo CL, Cohen SR, Richman JM, Rowlingson AJ. Efficacy of postoperative patient-controlled and continuous infusion epidural analgesia versus intravenous patient-controlled analgesia with opioids: a meta-analysis. Anesthesiology 1998; 89:1455-63

5. Md Abdul Hye, Khandakar Md Masud, Debasish Banik, Debabrata Banik, Fazlul Haque ANM, Akhtaruzzaman AKM. Intrathecal neostigmine for postoperative analgesia in caesarean section. Mymensing Medical Journal [Accepted for publication].

6. Akhtaruzzaman AKM, Agarwal AK, Iqbal KM. Role of Midazolam added to low dose hyperbaric Bupivacaine in sub-arachnoid block for lower uterine caesarean section-a comparative study. European Journal of Pain 2006; 10:1. 199.

7. Malleeswaran S, Panda N, Mathew P, Bagga $R$. A randomized study of magnesium sulphate as an adjuvant to intrathecal bupivacaine in patients with mild pre-eclampsia undergoing caesarean section. International Journal of Obstetric Anaesthesia 2010; 19: 161-166.

8. Buvanendran A, Sadegi M, Firazian A, Tabassomi F. Intrathecal magnesium prolongs fentanyl analgesia: a prospective, randomized controlled trail. Anesth Analg 2002; 95: 661-66. 